JOURNAL OF THE

AMERICAN MATHEMATICAL SOCIETY

Volume 16, Number 1, Pages 69-90

S 0894-0347(02)00404-6

Article electronically published on September 10, 2002

\title{
MEASURES INDUCED BY ANALYTIC FUNCTIONS AND A PROBLEM OF WALTER RUDIN
}

\author{
CARL SUNDBERG
}

\section{INTRODUCTION}

Let $H^{\infty}$ denote the set of bounded analytic functions in the unit disk

$$
U=\{z \in \mathbb{C}:|z|<1\}
$$

and let $H_{0}^{\infty}$ denote those $\varphi \in H^{\infty}$ for which $\varphi(0)=0$. Every $\varphi \in H^{\infty}$ has a nontangential limit at almost every point of $\partial U$, and the resulting function on $\partial U$ is measurable (for this and other basic facts about $H^{\infty}$ and related function spaces discussed in this paper, see, e.g., [D], [G] ). We denote the boundary function of $\varphi$ also by $\varphi$ and define the induced measure of $\varphi$ by the formula

$$
\mu_{\varphi}(E)=\frac{1}{2 \pi}\left|\left\{e^{i \theta} \in \partial U: \varphi\left(e^{i \theta}\right) \in E\right\}\right|
$$

for any measurable $E \subset \mathbb{C}$, where $|\cdot|$ denotes Lebesgue measure on $\partial U$. It is immediate that

$$
\int f(z) d \mu_{\varphi}(z)=\int f\left(\varphi\left(e^{i \theta}\right)\right) \frac{d \theta}{2 \pi}
$$

for any $f \in L^{1}\left(\mu_{\varphi}\right)$. In this paper we will always adopt the normalization $\varphi(0)=0$, i.e. $\varphi \in H_{0}^{\infty}$, and shall exclude the trivial case $\varphi \equiv 0$.

There is an important connection between induced measures and the classical Nevanlinna counting function $N_{\varphi}$ of $\varphi \in H_{0}^{\infty}$, defined by

$$
N_{\varphi}(w)=\sum_{\varphi(z)=w} \log \frac{1}{|z|},
$$

where the sum is understood to count multiplicities. To see this, write, for $w \in \mathbb{C}$,

$$
\varphi(z)-w=\Phi_{w}(z) B_{w}(z) S_{w}(z)
$$

where $\Phi_{w}$ is outer, $B_{w}$ is a Blaschke product, and $S_{w}$ is a singular inner function.

The zeros of $B_{w}$ are of course the roots of $\varphi(z)=w$, so

$$
\begin{aligned}
\int \log \left|\varphi\left(e^{i \theta}\right)-w\right| \frac{d \theta}{2 \pi} & =\int \log \left|\Phi_{w}\left(e^{i \theta}\right)\right| \frac{d \theta}{2 \pi} \\
& =\log \left|\Phi_{w}(0)\right| \\
& =\log |w|+\sum_{\varphi(z)=w} \log \frac{1}{|z|}+\log \frac{1}{\left|S_{w}(0)\right|} .
\end{aligned}
$$

Received by the editors May 5, 2000 and, in revised form, August 5, 2002.

2000 Mathematics Subject Classification. Primary 30D50.

Research supported in part by the National Science Foundation. 
By a theorem of Rudin $([\underline{\mathrm{R}}]), S_{w} \equiv 1$ for all $w$ off a set of capacity zero. By (1.2) and (1.3) we thus see that

$$
\int \log |z-w| d \mu_{\varphi}(z) \geq \log |w|+N_{\varphi}(w)
$$

for all $w \in \mathbb{C}$, with equality holding off a set of capacity zero.

Induced measures show up in a natural way in the study of composition operators. For $0<p<\infty$ we let $H^{p}$ denote the usual Hardy class of analytic functions in $U$ (see, e.g., [D], [G]). By the Littlewood Subordination Principle ([L]), if $\varphi \in H_{0}^{\infty}$ maps $U$ into $U$, then $f \circ \varphi \in H^{p}$ whenever $f \in H^{p}$, and in fact $\|f \circ \varphi\|_{H^{p}} \leq\|f\|_{H^{p}}$. We can thus define the composition operator $C_{\varphi}: H^{p} \rightarrow H^{p}$ by

$$
C_{\varphi}(f)=f \circ \varphi \text {. }
$$

By (1.2)

$$
\left\|C_{\varphi}(f)\right\|_{H^{p}}^{p}=\int|f(z)|^{p} d \mu_{\varphi}(z),
$$

so many questions concerning composition operators can be viewed as questions concerning induced measures. Here we mention only a result of Joel Shapiro $[\mathbf{S}]$ :

Theorem (J. Shapiro). The operator $C_{\varphi}: H^{p} \rightarrow H^{p}$ is compact if and only if $N_{\varphi}(w)=o(1-|w|)$.

A different necessary and sufficient condition can be formulated using induced measures. Recall that a positive measure $\mu$ on $U$ is said to be a Carleson measure if

$$
\mu\left(S_{h}\left(e^{i \theta_{0}}\right)\right)=O(h),
$$

where

$$
S_{h}\left(e^{i \theta_{0}}\right)=\left\{r e^{i \theta}: 1-h \leq r<1,\left|\theta-\theta_{0}\right| \leq h\right\} .
$$

It was shown by Carleson ([C1], C2]) that (1.6) is necessary and sufficient for the existence of a constant $C<\infty$ such that

$$
\int|f(z)|^{p} d \mu(z) \leq C\|f\|_{H^{p}}^{p}
$$

for all $f \in H^{p}$. It is immediate that this result continues to hold if $\mu$ is supported in $\operatorname{clos} U$ and the definition of $S_{h}\left(e^{i \theta_{0}}\right)$ is modified by allowing $r=1$. It is then easy to show using (1.6) that $C_{\varphi}$ is compact if and only if

$$
\mu_{\varphi}\left(S_{h}\left(e^{i \theta_{0}}\right)\right)=o(h) \text {. }
$$

One can thus prove Shapiro's theorem by directly showing that

$$
\int \log |z-w| d \mu_{\varphi}(z)-\log |w|=o(1-|w|)
$$

if and only if

$$
\mu_{\varphi}\left(S_{h}\left(e^{i \theta_{0}}\right)\right)=o(h) .
$$

In fact, although this point of view is not explicitly stated in Shapiro's paper, it is implicit in much of his approach.

It is natural to ask which measures $\mu$ can arise as induced measures. A necessary condition can be seen by (1.4) to be that the logarithmic potential of $\mu$ is bounded below by $\log |w|$. Another, coming from (1.2), is that $\int u(z) d \mu(z)=u(0)$ for any harmonic polynomial $u$. 
To obtain a third necessary condition, we consider the values that the logarithmic potential of an induced measure takes near 0 . For $\varphi \in H_{0}^{\infty}, \varphi \not \equiv 0$, write

$$
\varphi(z)=z^{n} g(z),
$$

where $g(0) \neq 0$. It is well known $([\mathbf{C}]$, Chap. 4, Theorem 7.4) that we can find $\varepsilon>0$ and $\delta>0$ making the following statements true:

If $0<|w|<\delta$, there exists exactly $n$ roots of $\varphi(z)=w$ in $[|z|<\varepsilon]$, all of them simple, and if $|z|<\varepsilon$, then $|g(z)|>\frac{1}{2}|g(0)|$.

Now let $0<|w|<\delta$ and let $z_{1}, \ldots, z_{n}$ be the roots of $\varphi(z)=w$ in $|z|<\varepsilon$. Then

$$
|w|=\left|z_{j}^{n} g\left(z_{j}\right)\right| \geq \frac{1}{2}\left|z_{j}\right|^{n}|g(0)|, \quad \text { so } \quad\left|z_{j}\right| \leq\left(\frac{2|w|}{|g(0)|}\right)^{1 / n}
$$

for $j=1, \ldots, n$. Thus by (1.4)

$$
\begin{aligned}
\int \log |z-w| d \mu_{\varphi}(z) & \geq \log |w|+\sum_{j=1}^{n} \log \frac{1}{\left|z_{j}\right|} \\
& \geq \log |w|+\log \frac{|g(0)|}{2|w|} \\
& =\log \frac{|g(0)|}{2} .
\end{aligned}
$$

We also have an estimate for $w=0$, namely

$$
\begin{aligned}
\int \log |z| d \mu_{\varphi}(z) & =\int \log \left|\varphi\left(e^{i \theta}\right)\right| \frac{d \theta}{2 \pi} \\
& =\int \log \left|g\left(e^{i \theta}\right)\right| \frac{d \theta}{2 \pi} \\
& \geq \log |g(0)| .
\end{aligned}
$$

Putting these observations together, we see that if $\mu=\mu_{\varphi}$ for some $\varphi \in H_{0}^{\infty}$, $\varphi \not \equiv 0$, then

a) $\int u(z) d \mu(z)=u(0)$ for any harmonic polynomial $u$,

b) $\int \log |z-w| d \mu(z) \geq \log |w|$ for all $w \in \mathbb{C}$, and

c) $\int \log |z-w| d \mu(z)$ is bounded below.

We now make the following

Conjecture. Suppose $\mu \geq 0$ is a compactly supported measure on $\mathbb{C}$ satisfying a), $b)$, and c). Then $\mu=\mu_{\varphi}$ for some $\varphi \in H_{0}^{\infty}$.

Remarks. 1) Taken together, a) and b) are equivalent to the statement

$$
\int v(z) d \mu(z) \geq v(0)
$$

for any function $v$ that is subharmonic on $\mathbb{C}$. However, it will be more convenient for us to keep these two conditions separate.

2) Suppose $\mu$ is a radial measure with a polar decomposition

$$
d \mu\left(r e^{i \theta}\right)=d w(r) \frac{d \theta}{2 \pi}
$$

where $w$ is a positive measure on $[0, \infty)$. It is easy to show that a) and b) are satisfied if $\|w\|=1$, and that $\mathrm{c}$ ) is satisfied if and only if $\log r \in L^{1}(w)$. In particular, if $\mu$ 
is defined to be one-half of normalized Lebesgue measure on $[|z|=1]$ and one-half of normalized Lebesgue measure on $[|z|=2]$, then $\mu$ satisfies a), b), and c).

The main results of this paper are the following two theorems, which may be seen as offering support for our conjecture.

Theorem 1. Let $\mu \geq 0$ be a compactly supported measure satisfying a) and b). Then there exist $\varphi_{n} \in H_{0}^{\infty}$ such that the support of $\mu_{\varphi_{n}}$ approaches the support of $\mu$, clos $\varphi_{n}(U)$ approaches the closure of the set where the logarithmic potential of $\mu$ exceeds $\log |z|$, and $\mu_{\varphi_{n}}$ approaches $\mu$ weakly. More precisely: If $V$ is an open set containing spt $\mu$ and $W$ is an open set containing the closure of the set $\left\{w: \int \log |w-z| d \mu(z)>\log |w|\right\}$, then $\operatorname{spt} \mu_{\varphi_{n}} \subset V$ and $\operatorname{clos} \varphi_{n}(U) \subset W$ for sufficiently high $n$, and

$$
\int f(z) d \mu_{\varphi_{n}}(z) \rightarrow \int f(z) d \mu(z)
$$

for any continuous function $f$.

Theorem 2. There exists a $\varphi \in H_{0}^{\infty}$ such that $\mu_{\varphi}$ is one-half of normalized Lebesgue measure on $[|z|=1]$ and one-half of normalized Lebesgue measure on $[|z|=2]$.

Theorem 2 answers a question posed by Walter Rudin. In a 1988 conference at the Mathematical Sciences Research Institute in Berkeley, California, Rudin noted that if $\varphi$ is an inner function for which $\varphi(0)=0$, then

$$
\int \varphi\left(e^{i \theta}\right)^{m} \overline{\varphi\left(e^{i \theta}\right)^{n}} \frac{d \theta}{2 \pi}=0 \quad \text { if } m \neq n,
$$

and asked if this condition implies that $\varphi$ is a constant multiple of an inner function. The function of Theorem 2 provides a counterexample, since by (1.2)

$$
\begin{aligned}
\int \varphi\left(e^{i \theta}\right)^{m} \overline{\varphi\left(e^{i \theta}\right)^{n}} \frac{d \theta}{2 \pi} & =\int z^{m} \bar{z}^{n} d \mu_{\varphi}(z) \\
& =\frac{1}{2} \int e^{i m \theta} e^{-i n \theta} \frac{d \theta}{2 \pi}+\frac{1}{2} \int\left(2^{m} e^{i m \theta} 2^{n} e^{-i n \theta}\right) \frac{d \theta}{2 \pi} \\
& =0 \quad \text { if } \quad m \neq n
\end{aligned}
$$

but $\varphi$ clearly cannot be a constant multiple of an inner function since the essential range of $|\varphi|$ on $\partial U$ is $\{1,2\}$. This argument was shown to me by John B. Conway, who in an unpublished manuscript showed that in fact (1.7) is equivalent to the rotational invariance of $\mu_{\varphi}$. A closely related result is due to Paul S. Bourdon, who showed in [B] that (1.7) is equivalent to the rotational invariance of $N \varphi$ modulo a set of measure zero. The connection between Conway's and Bourdon's result is of course given by (1.4) and the statement following it.

The rest of this paper is organized as follows. Section2 contains a review of some pertinent background and definitions, Section 3 is devoted to the proof of Theorem [1 and Section 4 to that of Theorem 2.

\section{Preliminaries}

In this section we briefly review some basic concepts connected with harmonic measures. Let $\Omega \subset \mathbb{C}$ be a bounded domain with smooth boundary. If $f \in C(\partial \Omega)$, then there is a unique function $u_{f} \in C(\cos \Omega)$ that is harmonic in $\Omega$ and agrees with $f$ on $\partial \Omega$ (see, e.g., $[\mathrm{Ke}]$, Chap. 11, $\mathrm{ABR}]$, Chap. 11, [C], Chap. 10, $\mathrm{F}$, Chap. 
1). For a point $a \in \Omega$ the map $f \mapsto u_{f}(a)$ is then a positive linear functional on $C(\partial \Omega)$ that takes 1 to 1 ; hence there is a probability measure $\omega=\omega_{a}$ such that $u_{f}(a)=\int_{\partial \Omega} f d \omega_{a}$ for all $f \in C(\partial \Omega)$. We refer to this measure as harmonic measure on $\partial \Omega$ with respect to $\Omega$, at $a$. This measure is in fact given by a smooth density $\rho=\rho_{a}$, so that $d \omega_{a}=\rho_{a} d m$, where $m$ is one-dimensional Lebesgue measure on $\partial \Omega$. If $E \subset \partial \Omega$ is a Borel set, then the function $u(z)=\omega_{z}(E)$ is a bounded harmonic function with nontangential limits equal to 1 a.e. $[m]$ on $E$ and 0 a.e. $[m]$ on $\partial \Omega \backslash E$. This fact gives us an alternate characterization of $\omega_{a}(E)$.

Harmonic measure can be studied by means of Green's functions. The Green's function of $\Omega$ with pole at $a$ is the unique function $G \in C(\operatorname{clos} \Omega \backslash\{a\})$ such that $G \equiv 0$ on $\partial \Omega$ and $G(z)-\log |z-a|$ extends to a harmonic function in $\Omega$. Then

$\rho=\rho_{a}$ is given by $\rho=\frac{1}{2 \pi} \frac{\partial G}{\partial n}$, where $\frac{\partial}{\partial n}$ is the directional derivative along the outward pointing unit normal to $\partial \Omega$.

We will also make use of the connection between harmonic measure and Brownian motion. For the definition and basic properties of Brownian motion, see, e.g., [PS], $[\mathrm{P}$. We will think of Brownian motion on $\mathbb{C}$ starting at $a$ simply as a probability measure $P$ on the set of continuous paths $X:[0, \infty) \rightarrow \mathbb{C}$ for which $X(0)=a$. With $\Omega$ as above let $\tau_{X}=\sup \{t: X([0, t]) \subset \Omega\}$. Then $\tau_{X}<\infty$ almost surely, and by a famous theorem of Kakutani, we have that $\omega_{a}(E)=P\left(X\left(\tau_{X}\right) \in E\right.$ ) (see $[\mathrm{K}],[\mathrm{P}]$.

We now discuss the connection between induced measures and harmonic measures, which will be of basic importance in this paper. By the Uniformization Theorem ( $\mathrm{AS}], \mathrm{Sp})$ there is an analytic covering map $\varphi$ of the unit disk $U$ onto $\Omega$ taking 0 to $a$. The nontangential limit function of $\varphi$ takes values a.e. in $\partial \Omega$. If $u \in C(\operatorname{clos} \Omega)$ is harmonic in $\Omega$, then $u \circ \varphi$ is a bounded harmonic function in $U$ with nontangential limit function $u \circ \varphi$.

Hence

$$
u(a)=u \circ \varphi(0)=\int u \circ \varphi\left(e^{i \theta}\right) \frac{d \theta}{2 \pi}=\int u(z) d \mu_{\varphi}(z),
$$

so we see that $\omega_{a}=\mu_{\varphi}$.

For the proof of Theorem 2 we will need to extend these ideas to certain Riemann surfaces. A finite bordered Riemann surface is a subset of a compact Riemann surface whose boundary consists of a finite number of analytic (or smooth) simple closed curves. With easy and obvious modifications most of the facts we have been discussing extend to these surfaces. In fact the surfaces we will use will all be constructed to have a natural projection $\pi$ to $\mathbb{C}$ via which we can get natural definitions of Lebesgue measure on the boundary and Brownian motion on the surface. The Uniformization Theorem in the form which we will need states that if a Riemann surface $\mathfrak{R}$ (not necessarily finite bordered) supports a nonconstant bounded analytic function and $a \in \mathfrak{R}$, then there is an analytic covering map $\varphi$ of $U$ onto $\Re$ taking 0 to $a$.

\section{Proof of Theorem 1}

Let $\mu \geq 1$ be a compactly supported measure on $\mathbb{C}$ satisfying

a) $\int u(z) d \mu(z)=u(0)$ for any harmonic polynomial $u$ and

b) $\int \log |z-w| d \mu(w) \geq \log |z|$ for all $z \in \mathbb{C}$. 
Let $K$ be the support of $\mu$ and $L$ be the closure of the set

$$
\left\{z \in \mathbb{C}: \int \log |z-w| d \mu(w)>\log |z|\right\} .
$$

It follows from a) that $L$ is compact, and we claim that $L$ is a connected set containing 0 . To prove this, note that if it were not true then there would exist a bounded connected open set $U$ with smooth boundary such that $0 \notin U, U \cap L=\varnothing$, and $\partial U \cap(L \cup\{0\})=\varnothing$. The function of $z$ given by

$$
\int \log |z-w| d \mu(w)-\log |z|
$$

would then be a nonnegative subharmonic function in $U$ that vanished on a neighborhood of $\partial U$. It would follow that this function vanished on all of $U$, which would contradict the definition of $L$ since $U \cap L \neq \varnothing$.

We are going to construct a sequence of bounded, smoothly bounded domains $\Omega_{n} \subset \mathbb{C}$ containing 0 such that $\partial \Omega_{n} \rightarrow K, \operatorname{clos} \Omega_{n} \rightarrow L$, and $\omega_{\Omega_{n}}$ approaches $\mu$ weakly, where $\omega_{\Omega_{n}}$ is harmonic measure with respect to $\Omega_{n}$, at 0 . As we have discussed in Section 2, if $\varphi_{n} \in H_{0}^{\infty}$ is a covering map of $\Omega_{n}$ such that $\varphi_{n}(0)=0$, then $\varphi_{n}$ will satisfy the conditions of the conclusion of the theorem. The domains $\Omega_{n}$ are obtained by setting $\varepsilon=\frac{1}{n}, n=1,2, \ldots$, in the following construction.

Fix an $\varepsilon>0$ and let

$$
\begin{aligned}
V & =\{z \in \mathbb{C}: \operatorname{dist}(z, K)<\varepsilon\}, \\
W & =\{z \in \mathbb{C}: \operatorname{dist}(z, L)<\varepsilon\} .
\end{aligned}
$$

If $\Gamma \subset \mathbb{C}$ is a simple closed curve, we denote by $\operatorname{Int} \Gamma$ the bounded component of $\mathbb{C} \backslash \Gamma$, and by Ext $\Gamma$ the unbounded component of $\mathbb{C} \backslash \Gamma$. There exist pairwise disjoint smooth simple closed curves $\Gamma_{0}, \ldots, \Gamma_{n}$ contained in $W \backslash L$ such that

$$
L \subset \operatorname{Int} \Gamma_{0} \cap \bigcap_{j=1}^{N} \operatorname{Ext} \Gamma_{j},
$$

each bounded component of $\mathbb{C} \backslash W$ is contained in $\bigcup_{j=1}^{N} \operatorname{Int} \Gamma_{j}$, each $\operatorname{Int} \Gamma_{j}$ for $j=1, \ldots, N$ contains a bounded component of $\mathbb{C} \backslash W$, and

$$
\operatorname{Int} \Gamma_{j} \cap \operatorname{Int} \Gamma_{k}=\varnothing \quad \text { for } \quad 1 \leq j, k \leq N, j \neq k
$$

(see e.g. [] ]); of course it may happen that $N=0$. Note that by a) the unbounded components of $\mathbb{C} \backslash K$ and $\mathbb{C} \backslash L$ coincide. By moving the curves $\Gamma_{0}, \ldots, \Gamma_{n}$ slightly we obtain pairwise disjoint smooth simple closed curves $\Gamma_{0}^{\prime}, \ldots, \Gamma_{N}^{\prime}$ satisfying the same conditions as $\Gamma_{0}, \ldots, \Gamma_{N}$ and such that

$$
\Gamma_{0}^{\prime} \subset \operatorname{Int} \Gamma_{0}
$$

and

$$
\Gamma_{j}^{\prime} \subset \operatorname{Ext} \Gamma_{j} \quad \text { for } \quad j=1, \ldots, N .
$$

Denote the space between $\Gamma_{j}$ and $\Gamma_{j}^{\prime}$ by $A_{j}$, i.e.

$$
\begin{aligned}
& A_{0}=\operatorname{Int} \Gamma_{0} \cap \operatorname{Ext} \Gamma_{0}^{\prime}, \\
& A_{j}=\operatorname{Ext} \Gamma_{j} \cap \operatorname{Int} \Gamma_{j}^{\prime} \quad \text { for } \quad j=1, \ldots, N .
\end{aligned}
$$

Let $\chi \geq 0$ be a rotationally symmetric continuous function supported in

$$
\left\{z \in \mathbb{C}:|z|<\operatorname{dist}\left(L, \Gamma_{0}^{\prime} \cup \cdots \cup \Gamma_{n}^{\prime}\right)\right\}
$$


and having integral 1. Then $\nu=\chi * \mu$ is a continuous function times area measure with support in $V$ such that the closure of

$$
\left\{z \in \mathbb{C}: \int \log |z-w| d \nu(w)>\log |z|\right\}
$$

is contained in Int $\Gamma_{0}^{\prime} \cap \bigcap_{j=1}^{N} \operatorname{Ext} \Gamma_{j}^{\prime}$. Using the mean value property for harmonic functions and the fact that $\log |z-w|$ is a subharmonic function of $z$, it is easy to show that $\nu$ satisfies a) and b).

For a large integer $n>\frac{1}{\sqrt{2} \varepsilon}$ to be determined in the course of the proof, we divide up $\mathbb{C}$ into small squares using the lines $x, y=\frac{j}{n}, j \in \mathbb{Z}$. Let $\left\{S_{k}\right\}$ denote those among these small squares having positive $\nu$-measure, and let $z_{k}$ be the center of $S_{k}$. If $n$ is sufficiently high, then

$$
S_{k} \subset \operatorname{Int} \Gamma_{0}^{\prime}
$$

and

$$
S_{k} \subset \bigcap_{j=1}^{N} \operatorname{Ext}_{j}^{\prime}
$$

for all $k$.

We need some estimates on the logarithmic potential of the measure $\sum_{j} \nu\left(S_{j}\right) \delta_{z j}$, on $\bigcup_{k} \partial S_{k}$. Fix $j$, and let $w \in S_{j}$. The function

$$
z \mapsto\left|\frac{z-z_{j}}{z-w}\right|
$$

attains its minimum on $\bigcup_{k} \partial S_{k}$ on $\partial S_{j}$ where it is clearly bounded below by $\frac{1}{2 \sqrt{2}}$. Hence if $z \in \bigcup_{k} \partial S_{k}$, then

$$
\nu\left(S_{j}\right) \log \left|z-z_{j}\right| \geq \int_{S_{j}} \log |z-w| d \nu(w)-\frac{3}{2} \log 2 \cdot \nu\left(S_{j}\right) .
$$

For $\delta>0$ a small number to be determined, if $\left|z-z_{j}\right|>\delta$ we can do better: then

$$
\left|\frac{z-z_{j}}{z-w}\right| \geq \frac{\delta}{\delta+\frac{1}{\sqrt{2} n}}
$$

for $w \in S_{j}$, so

$$
\nu\left(S_{j}\right) \log \left|z-z_{j}\right| \geq \int_{S_{j}} \log |z-w| d \nu(w)-\log \left(1+\frac{1}{\sqrt{2} \delta n}\right) \nu\left(S_{j}\right) .
$$

For $j=0, \ldots, N$ let $v_{j}$ be the function that is continuous on clos $A_{j}$ and harmonic in $A_{j}$ with boundary values

$$
\begin{array}{lll}
v_{j}=1 & \text { on } & \Gamma_{j}^{\prime}, \\
v_{j}=0 & \text { on } & \Gamma_{j},
\end{array}
$$

and set

$$
M=\sum_{j=0}^{N} \int_{\Gamma_{j}}\left|\frac{\partial v_{j}}{\partial n}\right| \frac{|d z|}{2 \pi} .
$$

Now pick $\delta$ so small that

$$
\nu(\{w:|w-z|<2 \delta\})<\frac{\varepsilon}{12 M \log 2} \quad \text { for all } z \in \mathbb{C} .
$$


Then, if $n>\frac{1}{\sqrt{2} \delta}$ is large enough,

$$
\log \left(1+\frac{1}{\sqrt{2} \delta n}\right)<\frac{\varepsilon}{8 M}
$$

Hence, by (3.1) and (3.2),

$$
\log |z|-\sum \nu\left(S_{j}\right) \log \left|z-z_{j}\right|<\log |z|-\int \log |z-w| d \nu(w)+\frac{\varepsilon}{4 M}
$$

for $z \in \bigcup_{j} \partial S_{j}$, so since $\nu$ satisfies b) we see that

$$
\log |z|-\sum \nu\left(S_{j}\right) \log \left|z-z_{j}\right|<\frac{\varepsilon}{4 M} \quad \text { for } z \in \bigcup_{j} \partial S_{j} .
$$

Define

$$
\tilde{\Omega}=\left\{z: \log |z|-\sum \nu\left(S_{j}\right) \log \left|z-z_{j}\right|-\frac{\varepsilon}{4 M}<0\right\} \cup\{\infty\} .
$$

From the definition of $S_{j}$ and the fact that $z_{j}$ is the center of $S_{j}, z_{j}$ must be of the form $\frac{k_{1}+\frac{1}{2}}{n}+i \frac{k_{2}+\frac{1}{2}}{n}$ for some integers $k_{1}, k_{2}$. Thus $z_{j} \neq 0$, so we see that $0 \in \tilde{\Omega}$. It is easy to see that $\tilde{\Omega}$ is a connected open subset of the Riemann sphere.

Denote the Green's function for $\tilde{\Omega}$ with pole at 0 by $G_{\tilde{\Omega}}$. Clearly

$$
G_{\tilde{\Omega}}(z)=\log |z|-\sum \nu\left(S_{j}\right) \log \left|z-z_{j}\right|-\frac{\varepsilon}{4 M} .
$$

From the above formula for $G_{\tilde{\Omega}}$ we see that $G_{\tilde{\Omega}}$ extends to a harmonic function in $\mathbb{C} \backslash\left(\left\{z_{j}\right\} \cup\{0\}\right)$ that goes to negative infinity at $\infty$, and $\partial \tilde{\Omega}$ is exactly the set of points at which it is 0 . It follows from (3.3) that $\partial \tilde{\Omega} \subset \bigcup S_{k}$. Fix $k$, let $-\eta=\sup _{\partial S_{k}} G_{\tilde{\Omega}}$, and let $U=\left\{z \in S_{k}: G_{\tilde{\Omega}}(z)>-\frac{1}{2} \delta\right\}$. Since $G_{\tilde{\Omega}}$ is harmonic in $S_{k} \backslash\left\{z_{k}\right\}$, it is easy to see that $U$ is connected and simply connected, and obviously $z_{k} \in U$. Let $f$ be a conformal map of $\mathbf{D}$ onto $U$ such that $f(0)=z_{k}$. Then $G_{\tilde{\Omega}} \circ f(z)=A \log \frac{1}{|z|}+B$ for some $A, B$; hence $\gamma_{k}=\partial \tilde{\Omega} \cap S_{k}=\left\{z \in S_{k}: G_{\tilde{\Omega}}(z)=0\right\}$ is a smooth simple closed curve such that $z_{k} \in \operatorname{Int} \gamma_{k}$.

Since

$$
\int_{\gamma} \frac{\partial}{\partial n_{z}} \log \left|z-z_{0}\right| \frac{|d z|}{2 \pi}= \begin{cases}1 & \text { if } z_{0} \in \operatorname{Int} \gamma \\ 0 & \text { if } z_{0} \in \operatorname{Ext} \gamma\end{cases}
$$

if $\gamma$ is any smooth simple closed curve, it is easy to see that

$$
\omega_{\tilde{\Omega}}\left(S_{j}\right)=\int_{\gamma_{j}} \frac{\partial G_{\tilde{\Omega}}(z)}{\partial n} \frac{|d z|}{2 \pi}=\nu\left(S_{j}\right) \quad \text { for all } j .
$$

Since $\operatorname{diam} S_{j}<\varepsilon$, this shows that $\omega_{\tilde{\Omega}}$ is close to $\nu$ in the appropriate sense, and of course $\nu$ is close to $\mu$. We must find a bounded domain $\Omega$ for which $\omega_{\Omega}$ is close to $\omega_{\tilde{\Omega}}$. To this end, we note that from (3.3) and the fact that

$$
\log |z|-\sum \nu\left(S_{j}\right) \log \left|z-z_{j}\right|-\frac{\varepsilon}{4 M}
$$

is a subharmonic function on the complement of $\bigcup S_{j}$ that is negative on $\bigcup \partial S_{j}$ and at $\infty$, we see that $\bigcup_{j=0}^{N} \operatorname{clos} A_{j} \subset \tilde{\Omega}$. Let

$$
\Omega=\tilde{\Omega} \cap \operatorname{Int} \Gamma_{0} \cap \bigcap_{k=1}^{N} \operatorname{Ext} \Gamma_{k} ;
$$


then $\partial \Omega=\bigcup \gamma_{j} \cup \bigcup \Gamma_{k}$. Since

$$
\log |z|-\int \log |z-w| d \nu(w) \equiv 0
$$

on each $A_{j}$, if $n$ is high enough we have

$$
|\log | z\left|-\sum \nu\left(S_{j}\right) \log \right| z-z_{j}||<\frac{\varepsilon}{4 M} \quad \text { for } z \in A_{j} .
$$

Let $v$ be a continuous function in $\operatorname{clos} \Omega$ that is harmonic in $\Omega$, with boundary values

$$
\begin{aligned}
& v(z)=-\left(\log |z|-\sum \nu\left(S_{j}\right) \log \left|z-z_{j}\right|-\frac{\varepsilon}{4 M}\right), \quad z \in \bigcup \Gamma_{k}, \\
& v(z)=0, \quad z \in \bigcup \gamma_{j} .
\end{aligned}
$$

Then the Green's function for $\Omega$ with pole at 0 is

$$
G_{\Omega}(z)=\log |z|-\sum \nu\left(S_{j}\right) \log \left|z-z_{j}\right|-\frac{\varepsilon}{4 M}+v(z) .
$$

By (3.5) we see that $|v(z)|<\frac{\varepsilon}{2 M}$ for all $z$, so by another application of (3.5), we see that

$$
-\frac{\varepsilon}{M}<G_{\Omega}(z)<0
$$

for $z \in A_{j}$. This clearly implies that

$$
0 \leq \frac{\partial G_{\Omega}}{\partial n}<\frac{\varepsilon}{M}\left|\frac{\partial v_{j}}{\partial n}\right| \quad \text { on } \Gamma_{j},
$$

so by the definition of $M$,

$$
\omega_{\Omega}\left(\bigcup \Gamma_{j}\right)=\sum_{j} \int_{\Gamma_{j}} \frac{\partial G_{\Omega}(z)}{\partial n} \frac{|d z|}{2 \pi}<\varepsilon .
$$

We now estimate $\left\|\omega_{\Omega}-\omega_{\tilde{\Omega}}\right\|$. To do this let $f$ be a continuous function on $\partial \Omega$, $\|f\|_{\infty} \leq 1$, let $\tilde{u}$ be continuous in $\operatorname{clos} \tilde{\Omega}$, harmonic in $\tilde{\Omega}$ with boundary values equal to $f$ on $\partial \tilde{\Omega}=\bigcup \gamma_{j}$, and let $u$ be continuous in $\operatorname{clos} \Omega$, harmonic in $\Omega$ with boundary values $f$. Then $u-\tilde{u}$ is continuous in $\operatorname{clos} \Omega$ and harmonic in $\Omega$ with boundary values 0 on $\partial \tilde{\Omega}$. Since $|u-\tilde{u}| \leq 2$ on $\bigcup \Gamma_{j}$, we see that

$$
\left|\int f d \omega_{\Omega}-\int f d \omega_{\tilde{\Omega}}\right|=|u(0)-\tilde{u}(0)| \leq 2 \omega_{\Omega}\left(\bigcup \Gamma_{j}\right)<2 \varepsilon .
$$

This of course shows that $\left\|\omega_{\Omega}-\omega_{\tilde{\Omega}}\right\|<2 \varepsilon$, and completes the proof.

\section{Proof of Theorem 2}

As in the proof of Theorem 1, we will produce our desired function $\varphi$ by means of a covering map, but in this case it will be a covering of a Riemann surface which we will construct. The basic step is a familiar construction which we will now describe. Let $\Omega_{1}, \Omega_{2}$ be two domains in $\mathbb{C}$ and $I$ a closed arc contained in their intersection. Let $\Omega_{2}^{\prime}$ be a copy of $\Omega_{2}$, disjoint from $\Omega_{2}$ and $\Omega_{1}$, and form a Riemann surface $\mathfrak{R}$ by gluing each side of $I$ on $\Omega_{1}$ to the opposite side on $\Omega_{2}^{\prime}$. We will refer to this construction as attaching $\Omega_{2}^{\prime}$ to $\Omega_{1}$ along $I$. By a slight abuse of terminology we will say that $\Omega_{1} \subset \mathfrak{R}$ and $\Omega_{2}^{\prime} \subset \mathfrak{R}$ (rather than, more accurately, $\Omega_{1} \backslash I \subset \mathfrak{R}$ and $\left.\Omega_{2}^{\prime} \backslash I \subset \mathfrak{R}\right)$. We will denote the points on a particular sheet by subscripts; e.g., if $0 \in \Omega_{1}$, we will denote by $0_{\Omega_{1}}$ the copy of 0 in $\mathfrak{R}$ on the sheet $\Omega_{1}$. 
Throughout this proof we will denote by $\mathbf{D}$ a fixed copy of the unit disk $[|z|<1]$ and by $\Delta$ the disk $[|z|<2]$. Suppose $\left\{I_{j}\right\}$ is an infinite collection of closed arcs contained in $\mathbf{D}$, clustering only on $\partial \mathbf{D}$. Let $\left\{\Delta_{j}\right\}$ be a family of copies of $\Delta$, pairwise disjoint and each disjoint from $\mathbf{D}$, and form a Riemann surface $\mathfrak{R}$ by attaching $\Delta_{j}$ to $\mathbf{D}$ along $I_{j}$, for each $j$. We have the natural projection $\pi: \mathfrak{R} \rightarrow \mathbb{C}$, with range $\Delta$.

The function $\pi$ is a nonconstant bounded analytic function on $\mathfrak{R}$; hence, as discussed in the last paragraph of Section 2 there is an analytic covering map $\chi$ from the unit disk, which in this context we will call $U$, onto $\mathfrak{R}$ such that $\chi(0)=0_{\mathbf{D}}$. Set $\varphi=\pi \circ \chi$.

The theorem will be proven by showing that the $\operatorname{arcs} I_{j}$ can be chosen so that $\mu_{\varphi}$ is as stated. To do this it will be convenient for us to use Brownian motion on $\mathfrak{R}$, which we can define simply by lifting Brownian motion on $\mathbb{C}$. To be more precise, let $X:[0, \infty) \rightarrow \mathbb{C}$ be a path such that $X(0)=0$ and $X(t)$ is not an endpoint of any of the $I_{j}$, for any $t \geq 0$. Then there is a unique lift $\tilde{X}$ of $X$ to a path on $\mathfrak{R}$ starting at $0_{\mathbf{D}}$, i.e. there is a unique path $\tilde{X}$ on $\mathfrak{R}$ such that $\tilde{X}(0)=0_{\mathbf{D}}$ and $\pi \circ \tilde{X}=X$. This lift exists for an interval in $t$ depending on $X$, which we will call $\left[0, \tau_{X}\right)$. Now suppose that instead of considering a single path $X$ we consider Brownian motion in $\mathbb{C}$ starting at 0 . For almost all such paths $X, X(t)$ is never an endpoint of one of the $I_{j}$ and $\tau_{X}<\infty$. The lifts of these paths give us a natural definition of Brownian motion on $\mathfrak{R}$ starting at $0_{\mathbf{D}}$. It is clear that $X\left(\tau_{X}\right)$ lies either on $\partial \mathbf{D}$ or $\partial \Delta$. If we attach $\partial \mathbf{D}$ to $\mathfrak{R}$ in the obvious way, we see that if $X\left(\tau_{X}\right) \in \partial \mathbf{D}$, then $\tilde{X}$ must hit $\partial \mathbf{D}$. We can now define a measure $\omega_{\mathfrak{R}}$ on $\partial \mathbf{D}$ by the following prescription: if $E \subset \partial D$ is measurable, then $\omega_{\mathfrak{R}}(E)$ is the probability that Brownian motion on $\mathfrak{R}$ starting at $0_{\mathbf{D}}$ will hit $E$. As the notation and the discussion in Section 2 suggest, $\omega_{\Re}$ can be thought of as harmonic measure on (part of) $\partial \Re$. We could in fact make this suggestion more precise by a careful definition of $\partial \Re$, but this would introduce technicalities that we wish to avoid.

Since analytic functions preserve Brownian motion (see, e.g., $[\mathrm{P}]$ ), the image under $\chi: U \rightarrow \mathfrak{R}$ of Brownian motion on $U$ starting at 0 is the Brownian motion on $\mathfrak{R}$ that we have been discussing (with a changed clock). Now suppose $X$ is a path starting at $0 \in U$ such that $\chi \circ X$ leaves $\mathfrak{R}$ at a point $w_{0} \in \partial \mathbf{D}$, at time $t_{0}$. By the properties of covering maps, $X\left(t_{0}\right)=z_{0} \in \partial U$, and we see that $\varphi\left(z_{0}\right)=$ $\varphi\left(X\left(t_{0}\right)\right)=\pi\left(w_{0}\right)=w_{0}$. Thus for $E \subset \partial \mathbf{D}$ measurable we see that $\omega_{\mathfrak{R}}(E)$ can be interpreted as the probability that a Brownian path in $U$ starting at 0 will hit $\partial U$ at a point $z$ for which $\varphi(z) \in E$. This of course shows that $\omega_{\mathfrak{R}}$ is the restriction of $\mu_{\varphi}$ to $\partial \mathbf{D}$.

We will show that the intervals $I_{j}$ can be chosen in such a way that

$$
d \omega_{\mathfrak{R}}\left(e^{i \theta}\right)=\frac{1}{2} \frac{d \theta}{2 \pi} .
$$

Obviously $\mu_{\varphi}$ is supported on $[|z|=1] \cup[|z|=2]$; since $\int u d \mu_{\varphi}=u(0)$ for all harmonic polynomials, it will then follow easily that $\mu_{\varphi}$ is as desired.

The choice of intervals will be made in infinitely many stages, and to reflect this we will change the notation a bit. We will pick numbers $\frac{1}{2}<s_{1}<s_{2}<\ldots$, with $s_{j} \nearrow 1$, and for each $j \geq 1$ a pairwise disjoint collection $\left\{I_{j k}\right\}_{k=1}^{n_{j}}$ of $\operatorname{arcs}$ on $\left[|z|=s_{j}\right]$. Set $\mathfrak{R}_{0}=\mathbf{D}$ and for $l \geq 1$ let $\mathfrak{R}_{l}$ be the Riemann surface obtained by attaching copies $\Delta_{j k}$ of $\Delta$ to $\mathbf{D}$ along $I_{j k}$ for $1 \leq j \leq l, 1 \leq k \leq n_{j}$. Let $\mathfrak{I}_{l}=\mathfrak{R}_{l-1} \backslash \bigcup_{k=1}^{n_{l}} I_{l k}$. 
The surface $\mathfrak{R}_{l}$ is a finite bordered Riemann surface, as discussed in Section 2 Let $\omega_{\mathfrak{R}_{l}}, \omega_{\mathfrak{I}_{l}}$ denote harmonic measure with respect to $\mathfrak{R}_{l}$, resp. $\mathfrak{I}_{l}$, at $0_{\mathbf{D}}$, restricted to $\partial \mathbf{D}$. These are given by smooth densities, i.e. there are smooth functions $\rho_{\mathfrak{R}_{l}}$, $\rho_{\mathfrak{I}_{l}}$ such that

$$
\begin{aligned}
d \omega_{\mathfrak{R}_{l}}\left(e^{i \theta}\right) & =\rho_{\mathfrak{R}_{l}}\left(e^{i \theta}\right) \frac{d \theta}{2 \pi}, \\
d \omega_{\mathfrak{I}_{l}}\left(e^{i \theta}\right) & =\rho_{\mathfrak{I}_{l}}\left(e^{i \theta}\right) \frac{d \theta}{2 \pi} .
\end{aligned}
$$

We are going to show (in Lemma 4.1) that we can pick a decreasing sequence of numbers $\varepsilon_{j}, 0<\varepsilon_{j}<\frac{1}{2^{j+1}}$, and the numbers $s_{j}$ and intervals $I_{j k}$ so that the following will be true:

$$
\begin{array}{ll}
\frac{\log \frac{1}{s_{j}}}{\log \frac{1}{s_{j-1}}}<\varepsilon_{j} & \text { for } j \geq 2, \\
\frac{\log \frac{2-s_{j}}{1-s_{j}}}{\log \frac{2-s_{j}}{\frac{1}{2}\left|I_{j k}\right|}<\varepsilon_{j}} & \text { for } j \geq 1 \text { and } 1 \leq k \leq n_{j}, \\
\rho_{\mathfrak{I}_{l}}\left(e^{i \theta}\right)>\frac{1}{2} & \text { for all } e^{i \theta}, l \geq 1, \\
\int \rho_{\mathfrak{R}_{l}}\left(e^{i \theta}\right) \frac{d \theta}{2 \pi}<\frac{1}{2}+\varepsilon_{l} & \text { for } l \geq 1 .
\end{array}
$$

(In (4.2) $\left|I_{j k}\right|$ is the length of $I_{j k}$.)

We will now show that if $\mathfrak{R}$ is formed as above with numbers $s_{j}$ and intervals $I_{j k}$ satisfying (4.1)-(4.4), then $\omega_{\mathfrak{R}}$ will be as desired. For $l=1,2, \ldots$ let $\mathfrak{R}_{\hat{l}}$ be the Riemann surface formed by attaching $\Delta_{j k}$ to $\mathbf{D}$ along $I_{j k}$ for all $j \neq l, 1 \leq k \leq n_{j}$. Fixing $l \geq 1$ for the moment, we let $q$ be the probability that Brownian motion on $R_{l-1}$ starting at $0_{\mathbf{D}}$ will hit the circle $\left[|z|=s_{l}\right]_{\mathbf{D}}$, and we let $p$ be the probability that it will hit $\left(\bigcup_{k=1}^{n_{l}} I_{l k}\right)_{\mathbf{D}}$. The harmonic measure of $[|z|=1]$ with respect to $\left[s_{l-1}<|z|<1\right]$ at a point $z$ such that $|z|=s_{l}$ is easily calculated to be $1-\frac{\log \frac{1}{s_{l}}}{\log \frac{1}{s_{l-1}}}$; this is of course the probability that a path in $\left[s_{l-1}<|z|<1\right]$ starting on $\left[|z|=s_{l}\right]$ will exit $\left[s_{l-1}<|z|<1\right]$ in the set $[|z|=1]$, and by (4.1) it is greater than $1-\varepsilon_{l}$. Hence

$$
q\left(1-\varepsilon_{l}\right)<\int \rho_{\Re_{l-1}}\left(e^{i \theta}\right) \frac{d \theta}{2 \pi}<\frac{1}{2}+\varepsilon_{l-1},
$$

where the right-hand inequality is (4.4). The probability that Brownian motion on $\mathfrak{R}_{l-1}$ starting at $0_{\mathbf{D}}$ will hit $\left[|z|=s_{l}\right]_{\mathbf{D}}$ but miss $\left(\bigcup_{k=1}^{n_{l}} I_{l k}\right)_{\mathbf{D}}$ is clearly $q-p$. On the other hand, this probability is greater than

$$
\int \rho_{\mathfrak{I}_{l}}\left(e^{i \theta}\right) \frac{d \theta}{2 \pi}
$$

which by (4.3) is greater than $\frac{1}{2}$. Hence

$$
q-p>\frac{1}{2}
$$

Combining (4.5) and (4.6), we see that

$$
p<\frac{2 \varepsilon_{l-1}+\varepsilon_{l}}{2\left(1-\varepsilon_{l}\right)} .
$$


With the same fixed $l$, let $\hat{p}$ be the probability that a Brownian path on $\mathfrak{R}_{\hat{l}}$ starting at $0_{\mathbf{D}}$ will hit $\left(\bigcup_{k=1}^{n_{l}} I_{l k}\right)_{\mathbf{D}}$. We wish to show that $\hat{p}<p+\varepsilon_{l+1}$. To this end, recalling the discussion at the beginning of this section, we consider Brownian paths on $\mathfrak{R}_{\hat{l}}$ and $\mathfrak{R}_{l-1}$ starting at $0_{\mathbf{D}}$ to be lifts of Brownian paths on $\mathbb{C}$ starting at 0 (neglecting the set of paths of probability zero that hit an endpoint of any of the intervals $I_{j k}$ ). We can then think of $\hat{p}-p$ as the probability that such a path has a lift to $\mathfrak{R}_{\hat{l}}$ that hits $\left(\bigcup_{k=1}^{n_{l}} I_{l k}\right)_{\mathbf{D}}$ before exiting $\mathfrak{R}_{\hat{l}}$, but has a lift to $\mathfrak{R}_{l-1}$ that exits $\mathfrak{R}_{l-1}$ before hitting $\left(\bigcup_{k=1}^{n_{l}} I_{l k}\right)_{\mathbf{D}}$. Consider such a path. In order for this to happen, the lift to $\mathfrak{R}_{\hat{l}}$ must hit a point $b \in \Delta_{j k}$, for some $j \geq l+1,1 \leq k \leq n_{j}$, that lies over $[|z|=1]$ before it hits $\left(\bigcup_{k=1}^{n_{l}} I_{l k}\right)_{\mathbf{D}}$, and then subsequently hit $\left(\bigcup_{k=1}^{n_{l}} I_{l k}\right)_{\mathbf{D}}$. But in order to do this it must travel from $b$ back to $\mathbf{D}$, and the only way it can do this is to pass back through $I_{j k}$. The probability it will do this is the harmonic measure of $I_{j k}$ with respect to $\Delta_{j k} \backslash I_{j k}$ at $b$, which is easily seen to be less than

$$
\frac{\log \frac{2+s_{j}}{1-s_{j}}}{\log \frac{2+s_{j}}{\frac{1}{2}\left|I_{j k}\right|}}
$$

by using the observations that, if $z_{j k}$ is the center of $I_{j k}$, then

$$
\left[\left|z-z_{j k}\right|<2+s_{j}\right] \supset \Delta_{j k}
$$

and

$$
\left|I_{j k}\right| \subset\left[\left|z-z_{j k}\right|<\frac{1}{2}\left|I_{j k}\right|\right] .
$$

By (4.2) we thus see that

$$
\hat{p}-p<\frac{\log \frac{2+s_{j}}{1-s_{j}}}{\log \frac{2+s_{j}}{\frac{1}{2}\left|I_{j k}\right|}}<\varepsilon_{j} \leq \varepsilon_{j+1},
$$

so by (4.7) we have

$$
\hat{p}<\varepsilon_{l+1}+\frac{2 \varepsilon_{l-1}+\varepsilon_{l}}{2\left(1-\varepsilon_{l}\right)} .
$$

Clearly, $\hat{p}$ is the probability that a Brownian path on $\mathfrak{R}$ starting at $0_{\mathbf{D}}$ ever hits $\bigcup_{k=1}^{n_{l}} \Delta_{l k}$, so this inequality with $l$ replaced by $l+1, l+2, \ldots$ shows that the probability that a Brownian path on $\mathfrak{R}$ starting at $0_{\mathbf{D}}$ ever hits $\bigcup_{j=l+1}^{\infty} \bigcup_{k=1}^{n_{j}} \Delta_{j k}$ is less than

$$
\sum_{j=l+1}^{\infty}\left[\varepsilon_{j+1}+\frac{2 \varepsilon_{j-1}+\varepsilon_{j}}{2\left(1-\varepsilon_{j}\right)}\right]
$$

Since $\varepsilon_{j}<\frac{1}{2^{j+1}}$, this sum is less than $\frac{1}{2^{l-1}}$. Now a path on $\mathfrak{R}$ starting at $0_{\mathbf{D}}$ that never hits $\bigcup_{j=l+1}^{\infty} \bigcup_{k=1}^{n_{j}} \Delta_{j k}$ is actually a path on $\mathfrak{R}_{l}$, so, using the Brownian motion picture of $\omega_{\Re_{l}}$, we see that we have shown that for $E \subset \partial \mathbf{D}$,

$$
\left|\omega_{\mathfrak{R}}(E)-\omega_{\mathfrak{R}_{l}}(E)\right|<\frac{1}{2^{l-1}} .
$$

It is clear that $\rho_{\mathfrak{R}_{l}}>\rho_{\mathfrak{I}_{l}}$, so by (4.3) we see that $\rho_{\mathfrak{R}_{l}}>\frac{1}{2}$. Thus, if $E \subset \partial \mathbf{D}$,

$$
\omega_{\mathfrak{R}_{l}}(E)=\int_{e^{i \theta} \in E} \rho_{\Re_{l}}\left(e^{i \theta}\right) \frac{d \theta}{2 \pi}>\frac{1}{2} \frac{|E|}{2 \pi} .
$$


Applying this to $F=\partial \mathbf{D} \backslash E$, we get

$$
\omega_{\mathfrak{R}_{l}}(E)<\omega_{\mathfrak{R}_{l}}(\partial \mathbf{D})-\frac{1}{2} \frac{|F|}{2 \pi}=\omega_{\mathfrak{R}_{l}}(\partial \mathbf{D})-\frac{1}{2}+\frac{1}{2} \frac{|E|}{2 \pi},
$$

so by (4.4) we see that

$$
\omega_{\mathfrak{R}_{l}}(E)<\varepsilon_{l}+\frac{1}{2} \frac{|E|}{2 \pi}<\frac{1}{2^{l+1}}+\frac{1}{2} \frac{|E|}{2 \pi} .
$$

Combining (4.9) and (4.10) with (4.8), we see that

$$
\left|\omega_{\mathfrak{R}}(E)-\frac{1}{2} \frac{|E|}{2 \pi}\right|<\frac{1}{2^{l-1}}+\frac{1}{2^{l+1}} \quad \text { for all } l,
$$

so $\omega_{\mathfrak{R}}(E)=\frac{1}{2} \frac{|E|}{2 \pi}$, as desired.

It remains to show that we can pick the $s_{j}$ and $I_{j k}$ so that (4.1)-(4.4) are satisfied. Changing notation again, we will show this inductively by proving the following:

Lemma 4.1. Suppose $\mathfrak{R}$ is a Riemann surface formed by attaching a finite number of copies of $\Delta$ to $\mathbf{D}$ along arcs all lying within $\left[|z| \leq r_{0}\right]$, and such that $\rho_{\mathfrak{R}}>\frac{1}{2}$ on $\partial \mathbf{D}$. Then, given $\varepsilon>0$, we can pick $s, r_{0}<s<1$, arbitrarily close to 1 , and a finite number of pairwise disjoint arcs $I_{j}$ of arbitrary small length lying on $[|z|=s]$, such that if $\tilde{\mathfrak{R}}$ is the Riemann surface formed by attaching pairwise disjoint copies $\Delta_{j}$ of $\Delta$ to $\mathfrak{R}$ along $I_{j}$, then

$$
\rho_{\mathfrak{R} \backslash \cup I_{j}}>\frac{1}{2} \quad \text { on } \quad \partial \mathbf{D}
$$

and

$$
\int \rho_{\tilde{\mathfrak{R}}}\left(e^{i \theta}\right) \frac{d \theta}{2 \pi}<\frac{1}{2}+\varepsilon .
$$

Proof. The proof of this lemma will be accomplished in two steps, in the first of which we will select some auxiliary $\operatorname{arcs}\left\{J_{k}\right\}$ along $[|z|=s]$, and in the second of which we define the $\operatorname{arcs} I_{j}$ as certain subarcs of the $J_{k}$.

Step 1 . We can pick $s, r_{0}<s<1$, arbitrarily close to 1 and a finite number of pairwise disjoint closed $\operatorname{arcs} J_{k}$ lying on $[|z|=s]_{\mathbf{D}}$ such that

$$
\rho_{\mathfrak{R} \backslash \cup J_{k}}>\frac{1}{2} \quad \text { on } \quad \partial \mathbf{D}
$$

and

$$
\int \rho_{\Re \backslash \cup J_{k}}\left(e^{i \theta}\right) \frac{d \theta}{2 \pi}<\frac{1}{2}+\varepsilon,
$$

where, as above, $\rho_{\mathfrak{R} \backslash \cup J_{k}}$ is the density with respect to normalized Lebesgue measure of harmonic measure with respect to $\Re \backslash \bigcup J_{k}$ at 0 , restricted to $\partial \mathbf{D}$.

If one thinks of the Brownian measure picture of harmonic measure, this step is fairly obvious intuitively; one selects the intervals in such a way as to "block" a suitable set of Brownian paths. The rigorous justification is, however, unfortunately long and complicated.

We need some definitions and lemmas.

Definition 4.1. For $0<s<1, A_{s}=[s<|z|<1]$.

Definition 4.2. For a function $f$ defined on $\partial \mathbf{D}$ and an $\operatorname{arc} K \subseteq \partial \mathbf{D}$,

$$
\operatorname{Var}_{K} f=\sup \{|f(z)-f(w)|: z, w \in K\} .
$$


Definition 4.3. If $K$ is a subarc of some circle $[|z|=r]$ and $\lambda>0$, then $\lambda K$ is the closed subarc of $[|z|=r]$ with the same center as $K$, and length $\lambda|K|$ (this assumes $\lambda|K|<2 \pi r)$.

Lemma 4.2. Suppose $u$ is a real-valued harmonic function in $A_{s}$, such that $|u(z)|$ $\leq 1$ for all $z \in A_{s}$ and $u(z) \rightarrow 0$ as $|z| \nearrow 1$. Then

$$
\left|\frac{\partial}{\partial \theta} \frac{\partial u(z)}{\partial n}\right|_{z=e^{i \theta}} \mid<\frac{4}{(1-s)^{2}} .
$$

Proof. Let $\tilde{A}_{s}=\left[s<|z|<\frac{1}{s}\right]$ and define $\tilde{u}$ in $\tilde{A}_{s}$ by

$$
\tilde{u}(z)= \begin{cases}u(z) & s<|z|<1, \\ 0 & |z|=1, \\ -u\left(\frac{1}{\bar{z}}\right) & 1<|z|<\frac{1}{s} .\end{cases}
$$

Then $\tilde{u}$ is harmonic in $\tilde{A}_{s}$, and of course $|\tilde{u}(z)| \leq 1$ for all $z \in \tilde{A}_{s}$. Hence, if $\left|z_{0}\right|=1$, then $\tilde{u}$ is harmonic in ||$\left.z-z_{0} \mid<1-s\right]$ and has modulus bounded by 1 there. Now if $v$ is harmonic in $[|z|<1]$ and has modulus bounded by 1 there, then it is easy to calculate that $\left|\frac{\partial^{2} v}{\partial x \partial y}\right|_{x=y=0} \mid \leq 4$; translating and rescaling this estimate proves the lemma.

Lemma 4.3. Suppose $0<r<s<1,0 \leq \delta<1, n$ is a positive integer, and

$$
E=\left\{s e^{i \theta}: \frac{2 \pi k}{n} \leq \theta \leq \frac{2 \pi}{n}(k+\delta) \text { for some } k \in\{0,1, \ldots, n-1\}\right\},
$$

and that $u$ is the continuous function on $\operatorname{clos} A_{r}$ that is harmonic in $A_{r} \backslash E$ and with boundary values

$$
\begin{array}{lll}
u=1 & \text { on } & {[|z|=r],} \\
u=0 & \text { on } & E \cup[|z|=1] .
\end{array}
$$

Then

$$
\operatorname{Var}_{[|z|=1]} \frac{\partial u}{\partial n} \leq \frac{2 \pi}{n} \frac{4}{(1-s)^{2}} .
$$

Proof. This follows from Lemma 4.1 and the obvious fact that $u\left(e^{2 \pi i / n} z\right)=u(z)$.

Lemma 4.4. Suppose $K$ is a closed proper subarc of $\partial \mathbf{D}, 0<\lambda<1, \frac{1}{2}<r<$ 1 is such that $1-r<\frac{1}{8}(1-\lambda)|K|$, and $a, b>0$. Let $R_{K, r}$ be the interior of $\left\{t e^{i \theta}: r<t<1, e^{i \theta} \in K\right\}$ and let $E \subset \operatorname{clos} R_{K, r}$ consist of finitely many closed subarcs of $[|z|=s]$, where $r<s<1$. Suppose $u$ is a real-valued continuous function on $\operatorname{clos} R_{K, r}$ that is harmonic in $R_{K, r} \backslash E$ and such that

$$
\begin{array}{rlrl}
u & =0 & & \text { on } E \cup K, \\
|u| \leq a \log \frac{1}{r} & & \text { on }[|z|=r], \\
|u| \leq b \log \frac{1}{r} & & \text { on the other two sides of } R_{K, r} .
\end{array}
$$

Then on $\lambda K$ we have the estimate

$$
\left|\frac{\partial u}{\partial n}\right| \leq 4 \log 2 \cdot\left[a+b \kappa\left(\frac{4 \log \frac{1}{r}}{(1-\lambda)|K|}\right)\right],
$$


where $\kappa$ is a function such that $\kappa(h) \searrow 0$ as $h \searrow 0$.

Proof. Write $u=u_{+}-u_{-}$, where $u_{ \pm}$are continuous on clos $R_{K, r}$ and harmonic in $R_{K, r} \backslash E$, and such that

$$
\begin{array}{ll}
u_{ \pm} \geq 0 & \text { in } R_{K, r}, \\
u_{ \pm}=0 & \text { on } E \cup K, \\
u_{ \pm} \leq a \log \frac{1}{r} & \text { on }[|z|=r], \\
u_{ \pm} \leq b \log \frac{1}{r} & \text { on the other two sides of } R_{K, r} .
\end{array}
$$

Let $v$ be the bounded harmonic function on $R_{K, r}$ with boundary values

$$
\begin{array}{lll}
v=a \log \frac{1}{r} & & \text { on }[|z|=r], \\
v=0 & \text { on }[|z|=1], \\
v=b \log \frac{1}{r} & & \text { on the other sides of } R_{K, r},
\end{array}
$$

and extend $v$ to $\tilde{v}$ on $\tilde{R}_{K, r}$, the interior of

$$
\left\{t e^{i \theta}: e^{i \theta} \in K, r<t<\frac{1}{r}\right\}
$$

by defining $\tilde{v}(z)=-v\left(\frac{1}{\bar{z}}\right)$ for $\frac{1}{\bar{z}} \in R_{K, r}$.

Suppose $z_{0} \in \lambda K$ and $\left|z-z_{0}\right|<1-r$. We wish to estimate $\tilde{v}(z)$. By our conditions relating $\lambda, r$, and $|K|$ we see that

$$
z \in S=\left\{t e^{i \theta}: r<t<\frac{1}{r}, e^{i \theta} \in \frac{1}{2}(1+\lambda) K\right\} .
$$

Using a conformal mapping of $\tilde{R}_{K, r}$ onto a rectangle, one sees that the harmonic measure of the radial sides of $\tilde{R}_{K, r}$ with respect to $\tilde{R}_{K, r}$ at $z$ is less than

$$
\kappa\left(\frac{4 \log \frac{1}{r}}{(1-\lambda)|K|}\right),
$$

where $\kappa(h)$ is defined as follows. Let $R_{h}$ be a rectangle with horizontal dimension 1 and vertical dimension $h$. Then $\kappa(h)$ is the harmonic measure of the vertical sides of $R_{h}$, with respect to $R_{h}$, at the center of $R_{h}$.

Thus in the disk $\left[\left|z-z_{0}\right|<1-r\right]$ we have the estimate

$$
|\tilde{v}(z)|<a \log \frac{1}{r}+b \log \frac{1}{r} \cdot \kappa\left(\frac{4 \log \frac{1}{r}}{(1-\lambda)|K|}\right) .
$$

It is easy to show that the gradient at the origin of a real-valued harmonic function in $[|z|<1]$, whose absolute value is bounded by 1 , is bounded by 2 (in fact, with a little more work one can show it is bounded by $\left.\frac{4}{\pi}\right)$; rescaling this to the disk $\left[\left|z-z_{0}\right|<1-r\right]$, we see that

$$
\begin{aligned}
\left|\frac{\partial v(z)}{\partial n}\right|_{z=z_{0}}|=| \nabla \tilde{v}(z) \mid & \leq 2\left[a+b \kappa\left(\frac{4 \log \frac{1}{r}}{(1-\lambda)|K|}\right)\right] \frac{\log \frac{1}{r}}{1-r} \\
& <4 \log 2 \cdot\left[a+b \kappa\left(\frac{4 \log \frac{1}{r}}{(1-\lambda)|K|}\right)\right] .
\end{aligned}
$$


Since $0 \leq u_{ \pm} \leq v$, it follows that

$$
\left.\left.\left.\left|\frac{\partial u(z)}{\partial n}\right|_{z=z_{0}}|=| \frac{\partial u_{ \pm}(z)}{\partial n}\right|_{z=z_{0}}|-| \frac{\partial u_{-}(z)}{\partial n}\right|_{z=z_{0}}|\leq| \frac{\partial v(z)}{\partial n}\right|_{z=z_{0}} \mid,
$$

and the lemma follows.

We now proceed with Step 1. Our main tool will be estimates on Green's functions of various surfaces with poles at $0_{\mathbf{D}}$, which we will denote by $G_{(\cdot)}$, where (.) will be the symbol for the surface. So, in particular,

$$
\rho_{\mathfrak{R}}=\frac{\partial G_{\mathfrak{R}}}{\partial n} \quad \text { on } \partial \mathbf{D} \text {. }
$$

It is easy to see, using the Brownian motion picture of harmonic measure, that

$$
\rho_{\mathfrak{R}} \leq 1 \quad \text { on } \partial \mathbf{D} .
$$

We may assume that $\varepsilon<\min _{\partial \mathbf{D}} \rho_{\Re}-\frac{1}{2}$ and $\varepsilon<\frac{1}{75}$. Let $\eta$ be a positive number such that

$$
1-\frac{1}{3} \varepsilon<\eta^{2}<1
$$

Divide $\partial \mathbf{D}$ into adjacent closed $\operatorname{arcs} K_{l}$, all of the same length $|K|$, so small that

$$
\operatorname{Var}_{K_{l} \cup K_{l^{\prime}}} \rho_{\Re}<\varepsilon^{2} \text { for any two adjacent } \operatorname{arcs} K_{l} \text { and } K_{l^{\prime}} \text {, }
$$

then pick $r$ between 0 and 1 , so close to 1 that

$$
1-r<\frac{1}{8}\left(\eta-\eta^{2}\right)|K|
$$

and

$$
\kappa\left(\frac{4 \log \frac{1}{r}}{\left(\eta-\eta^{2}\right)|K|}\right)<\varepsilon^{2},
$$

where $\kappa$ is as in Lemma 4.4 and so that

$$
\left|G_{\mathfrak{R}}\left(t e^{i \theta}\right)-\rho_{\mathfrak{R}}\left(e^{i \theta}\right) \log t\right|<\varepsilon^{2} \log \frac{1}{t} \quad \text { for all } e^{i \theta} \text { and } r \leq t<1 .
$$

For $s$ between $r$ and 1, define

$$
\mathfrak{R}_{s}=\mathfrak{R} \backslash[s \leq|z|<1]_{\mathbf{D}},
$$

then pick $s>\frac{1}{2}(1+r)$ so close to 1 that

$$
G_{\Re}\left(r e^{i \theta}\right)<G_{\mathfrak{R}_{s}}\left(r e^{i \theta}\right)<G_{\mathfrak{R}}\left(r e^{i \theta}\right)+\varepsilon^{2} \log \frac{1}{r} \quad \text { for all } e^{i \theta} .
$$

It will then follow that if $E$ is any finite collection of pairwise disjoint closed subarcs of $[|z|=s]_{\mathbf{D}}$, then

$$
\left|G_{\mathfrak{R}}\left(r e^{i \theta}\right)-G_{\mathfrak{R} \backslash E}\left(r e^{i \theta}\right)\right|<\varepsilon^{2} \log \frac{1}{r} \quad \text { for all } e^{i \theta} .
$$

Of course it will also be true that $\rho_{\mathfrak{R} \backslash E} \leq \rho_{\mathfrak{R}}$ on $\partial \mathbf{D}$.

For each $l$, set

$$
\rho_{l}=\min _{K_{l}} \rho_{\Re} .
$$

With an integer $n>\frac{8 \pi}{\varepsilon^{2}(1-s)^{2}}$, define, for $0<\delta<1$,

$$
E_{\delta}=\left\{s e^{i \theta}: \frac{2 \pi k}{n} \leq \theta \leq \frac{2 \pi(k+\delta)}{n} \text { for some } k \in\{0,1, \ldots, n-1\}\right\} .
$$


For $\mu>0$, define $u_{\delta, \mu}$ to be the bounded harmonic function in $A_{r} \backslash E_{\delta}$ with boundary values

$$
\begin{aligned}
& u_{\delta, \mu}=-\mu \log \frac{1}{r} \quad \text { on }[|z|=r] \\
& u_{\delta, \mu}=0 \quad \text { on }[|z|=1] \cup E_{\delta} \text {. }
\end{aligned}
$$

As a function of $\delta, \min _{[|z|=1]} \frac{\partial u_{\delta, \mu}}{\partial n}$ is continuous and takes the values $\mu$ at $\delta=0^{+}$ and 0 at $\delta=1^{-}$. Since $\rho_{l}>\frac{1}{2}+\varepsilon$, we see that for each $l$ there exists a $\delta_{l}$ between 0 and 1 such that

$$
\min _{[|z|=1]} \frac{\partial u_{\delta_{l}, \rho_{l}}}{\partial n}=\frac{1}{2}+\frac{1}{3} \varepsilon .
$$

We now define the set $E$ as follows:

$$
E=\bigcup_{l}\left\{s e^{i \theta} \in E_{\delta_{l}}: e^{i \theta} \in \eta K_{l}\right\} .
$$

We can clearly write $E=\bigcup J_{k}$, where $\left\{J_{k}\right\}$ is as described in the statement of Step 1 , and we will now show that (4.13) and (4.14) are satisfied.

We define, for every $l, u_{l}$ to be the bounded harmonic function in $A_{r} \backslash E_{\delta_{l}}$ with boundary values

$$
\begin{array}{ll}
u_{l}=-\rho_{l} \log \frac{1}{r} & \text { on }[|z|=r], \\
u_{l}=0 & \text { on }[|z|=1] \cup E_{\delta_{l}} .
\end{array}
$$

By Lemma 4.3 and the choice of $n$,

$$
\operatorname{Var}_{[|z|=1]} \frac{\partial u_{l}}{\partial n}<\varepsilon^{2}
$$

and by definition of $\delta_{l}$

$$
\min _{[|z|=1]} \frac{\partial u_{l}}{\partial n}=\frac{1}{2}+\frac{1}{3} \varepsilon .
$$

It follows from (4.16), (4.19), and (4.20) that

$$
\left|G_{\mathfrak{R} \backslash E}\left(r e^{i \theta}\right)-u_{l}\left(r e^{i \theta}\right)\right|<3 \varepsilon^{2} \log \frac{1}{r} \quad \text { for } e^{i \theta} \in K_{l} \cup K_{l^{\prime}},
$$

where $K_{l^{\prime}}$ is adjacent to $K_{l}$.

From (4.24), (4.17), and (4.19) together with the facts that $\rho_{\mathfrak{R}} \leq 1$ and $\left|G_{\mathfrak{R} \backslash E}\right| \leq$ $\left|G_{\mathfrak{R}}\right|$ it follows that we can now apply Lemma 4.4 with the role of

- $K$ played by $\eta K_{l}$,

- that of $\lambda$ played by $\eta$,

- that of $u$ played by $G_{\Re \backslash E}-u_{l}$,

- that of $E$ played by $E_{\delta_{l}}$,

- that of $a$ played by $3 \varepsilon^{2}$,

- and that of $b$ played by $2+\varepsilon^{2}$. 
By (4.18), (4.22), and (4.23) we thus see that, on $\eta^{2} K_{l}$,

$$
\begin{aligned}
\left|\rho_{\mathfrak{R} \backslash E}-\left(\frac{1}{2}+\frac{1}{3} \varepsilon\right)\right| & =\left|\frac{\partial G_{\mathfrak{R} \backslash E}}{\partial n}-\left(\frac{1}{2}+\frac{1}{3} \varepsilon\right)\right| \\
& \leq\left|\frac{\partial G_{\mathfrak{R} \backslash E}}{\partial n}-\frac{\partial u_{l}}{\partial n}\right|+\left|\frac{\partial u_{l}}{\partial n}-\left(\frac{1}{2}+\frac{1}{3} \varepsilon\right)\right| \\
& <4 \log 2 \cdot\left[3 \varepsilon^{2}+\left(2+\varepsilon^{2}\right) \kappa\left(\frac{4 \log \frac{1}{r}}{\left(\eta-\eta^{2}\right)|K|}\right)\right]+\varepsilon^{2} \\
& <25 \varepsilon^{2} .
\end{aligned}
$$

Since $\varepsilon<\frac{1}{75}, 1-\eta^{2}<\frac{1}{3} \varepsilon$, and $\rho_{\mathfrak{R} \backslash E} \leq 1$, it follows that (4.14) is satisfied.

It remains to be shown that (4.13) is satisfied. With $R_{(2-\eta) K_{l}, r}$ defined as in the statement of Lemma 4.4, define $v_{l}$ to be the bounded harmonic function in $R_{(2-\eta) K_{l}, r} \backslash E$ with boundary values

$$
\begin{array}{lll}
v_{l}=-\rho_{l} \log \frac{1}{|z|} & & \text { on the radial sides of } R_{(2-\eta) K_{l}, r}, \\
v_{l}=-\rho_{l} \log \frac{1}{r} & & \text { on }[|z|=r], \\
v_{l}=0 & \text { on }\left(E \cap R_{(2-\eta) K_{l}, r}\right) \cup(2-\eta) K_{l} .
\end{array}
$$

One easily shows that

$$
E \cap R_{(2-\eta) K_{l}, r}=E \cap R_{\eta K_{l}, r}
$$

and it follows that $v_{l} \leq u_{l} \leq 0$ on $R_{(2-\eta) K_{l}, r}$, so

$$
\frac{\partial v_{l}}{\partial n} \geq \frac{\partial u_{l}}{\partial n} \geq 0 \quad \text { on } \quad(2-\eta) K_{l}
$$

and so by (4.23)

$$
\frac{\partial v_{l}}{\partial n} \geq \frac{1}{2}+\frac{1}{3} \varepsilon \quad \text { on } \quad(2-\eta) K_{l} .
$$

It follows from (4.16), (4.19), and (4.20) that

$$
\left|G_{\mathfrak{R} \backslash E}\left(r e^{i \theta}\right)-v_{l}\left(r e^{i \theta}\right)\right|<3 \varepsilon^{2} \log \frac{1}{r} \quad \text { for } e^{i \theta} \in(2-\eta) K_{l} .
$$

From (4.26) and (4.19) together with the facts that $\rho_{\mathfrak{R}} \leq 1$ and $\left|G_{\mathfrak{R} \backslash E}\right| \leq\left|G_{\mathfrak{R}}\right|$ it follows that we can apply Lemma 4.4 with the role of

- $K$ played by $(2-\eta) K_{l}$,

- that of $\lambda$ played by $\frac{1}{2-\eta}$,

- that of $u$ played by $G_{\mathfrak{R} \backslash E}-v_{l}$,

- that of $E$ played by $E$,

- that of $a$ played by $3 \varepsilon^{2}$,

- and that of $b$ played by $2+\varepsilon^{2}$.

By (4.18) we thus see that

$$
\begin{aligned}
\left|\rho_{\Re \backslash E}-\frac{\partial v_{l}}{\partial n}\right| & =\left|\frac{\partial G_{\Re \backslash E}}{\partial n}-\frac{\partial v_{l}}{\partial n}\right| \\
& \leq 4 \log 2 \cdot\left[3 \varepsilon^{2}+\left(2+\varepsilon^{2}\right) \kappa\left(\frac{4 \log \frac{1}{r}}{(1-\eta)\left|K_{l}\right|}\right)\right] \\
& <24 \varepsilon^{2} \quad \text { on } K_{l} .
\end{aligned}
$$


Since $\varepsilon<\frac{1}{75}$, 4.13) follows from this and (4.25). This completes Step 1.

Step 2. Let $s$ and $\left\{J_{k}\right\}$ be as in Step 1, and set

$$
\tilde{\varepsilon}=\frac{1}{2}+\varepsilon-\int \rho_{\mathfrak{R} \backslash \cup J_{k}}\left(e^{i \theta}\right) \frac{d \theta}{2 \pi}>0 .
$$

With $N$ a positive integer to be chosen later, we divide each $J_{k}$ into $2 N+1$ subarcs of length $\frac{1}{2 N+1}\left|J_{k}\right|$, labeled sequentially as

$$
I_{k, 1}, \ldots, I_{k, 2 N+1} \text {. }
$$

For all $k$ and for $j=0,1, \ldots, N$ we attach a copy $\Delta_{k j}$ of $\Delta$ to $\mathfrak{R}$ along $I_{k, 2 j+1}$ to get a Riemann surface which we will call $\tilde{\mathfrak{R}}$. Clearly

$$
\rho_{\tilde{R}} \geq \rho_{\mathfrak{R} \backslash \cup J_{k}}>\frac{1}{2} \quad \text { on } \partial \mathbf{D} \text {. }
$$

We will show that if $N$ is big enough, then

$$
\int \rho_{\tilde{\mathfrak{R}}}\left(e^{i \theta}\right) \frac{d \theta}{2 \pi}<\int \rho_{\mathfrak{R} \backslash \cup J_{k}}\left(e^{i \theta}\right) \frac{d \theta}{2 \pi}+\tilde{\varepsilon}=\frac{1}{2}+\varepsilon,
$$

which will complete the proof.

We need to define some auxiliary sets. For each $k$ let $D_{k} \subset \mathbf{D}$ be an open disk symmetric with respect to the reflection $z \mapsto \frac{s^{2}}{\bar{z}}$ in the circle $[|z|=s]$, with center on the same radius as the center of $J_{k}$, and such that

$$
\begin{gathered}
\operatorname{clos} D_{k} \subset\left[r_{0}<|z|<1\right]_{\mathbf{D}}, \\
J_{k} \subset D_{k},
\end{gathered}
$$

and $\operatorname{clos} D_{k} \cap \operatorname{clos} D_{k^{\prime}}=\varnothing$ if $k \neq k^{\prime}$.

This is possible since $s>\frac{1}{2}(1+r)$ and $\left|J_{k}\right|<10^{-5}(1-s)$, as seen from the choice of $n$ in Step 1.

For a small $\delta>0$ to be determined in the course of the proof, let, for each $k$,

$$
J_{k}^{-} \subset[|z|=s-\delta]_{\mathbf{D}} \subset \mathfrak{R}
$$

and

$$
J_{k}^{+} \subset\left[|z|=s+\frac{s}{s-\delta} \delta\right]_{\mathbf{D}} \subset \mathfrak{R}
$$

be closed arcs that project radially onto $J_{k}$; note that $J_{k}^{+}$is the reflection of $J_{k}^{-}$ through $[|z|=s]$.

If $\eta$ is between 0 and 1 and close enough to 1 , and $\delta$ is small enough, then the following will be true:

$$
\left|\rho_{\Re \backslash \cup\left(\eta^{2} J_{k}^{+} \cup \eta^{2} J_{k}^{-}\right)}\left(e^{i \theta}\right)-\rho_{\mathfrak{R} \backslash \cup J_{k}}\left(e^{i \theta}\right)\right|<\frac{1}{3} \tilde{\varepsilon} \quad \text { for all } e^{i \theta} .
$$

Because of (4.28), (4.27) will be a consequence of

$$
\int \rho_{\tilde{R}}\left(e^{i \theta}\right) \frac{d \theta}{2 \pi}<\int \rho_{\mathfrak{R} \backslash \cup\left(\eta^{2} J_{k}^{+} \cup \eta^{2} J_{k}^{-}\right)}\left(e^{i \theta}\right) \frac{d \theta}{2 \pi}+\frac{2}{3} \tilde{\varepsilon} .
$$

We can construct Brownian motion on $\tilde{\mathfrak{R}}$ starting at an arbitrary point on $\mathbf{D}$ just as we did above for Brownian motion on $\tilde{\mathfrak{R}}$ starting at $0_{\mathbf{D}}$. If one then thinks of the Brownian motion description of harmonic measures, it is evident that (4.29) will be a consequence of:

The probability that a Brownian path on $\tilde{\mathfrak{R}}$ starting on $\bigcup\left(\eta^{2} J_{k}^{+} \cup \eta^{2} J_{k}^{-}\right)$will hit $\partial \mathbf{D}$ is less than $\frac{2}{3} \tilde{\varepsilon}$. 
In our proof of (4.30) we will concentrate on the behavior of paths near a single $\operatorname{arc} J_{k}$. For each $k$ let $\Re_{k}^{\prime}$ be the part of $\tilde{\Re}$ formed by joining each $\Delta_{k j}$ to $D_{k}$ along $I_{k, 2 j+1}$ for $j=0,1, \ldots, N$. We will show that if $\delta$ is small enough and $N$ is large enough, then the following will be true:

The probability that a Brownian path on $\mathfrak{R}_{k}^{\prime}$ starting on $\eta^{2} J_{k}^{+} \cup$ $\eta^{2} J_{k}^{-}$will hit $\bigcup_{j=1}^{N} \partial \Delta_{k j}$ is greater than $1-\frac{2}{3} \tilde{\varepsilon}$.

Clearly (4.31) implies (4.30).

Since (4.31) just involves a single $k$, we may simplify our notation a bit by dropping the index $k$, e.g. we write $\mathfrak{R}^{\prime}$ for $\mathfrak{R}_{k}^{\prime}$.

Choose an integer $m$ so large that

$$
\left(\frac{5}{6}\right)^{m}<\frac{1}{6} \tilde{\varepsilon}
$$

and

$$
\operatorname{dist}\left(J^{+} \cup J^{-}, \partial D\right)>\frac{\eta-\eta^{2}}{8 m}|J|,
$$

then choose a number $p$ between 0 and 1 , so close to 1 that

$$
p^{2 m}>1-\frac{1}{6} \tilde{\varepsilon} .
$$

Let $\Psi$ be a Möbius transformation that takes $D\left(=D_{k}\right)$ to the unit disk and $J$ to a real interval symmetric about 0 . For $z \in D$ let

$$
D_{z}=\Psi^{-1}(\{w:|w-\Psi(z)|<\sigma\}),
$$

where $\sigma>0$ is smaller than all of the following:

(i) $\frac{1}{2 m} \operatorname{dist}\left(\Psi\left(\eta^{2} J^{+} \cup \eta^{2} J^{-}\right), \partial \Psi\left(\eta J^{+} \cup \eta J^{-}\right)\right)$,

(ii) $\operatorname{dist}\left(\Psi\left(\eta J^{+} \cup \eta J^{-}\right), \partial \Psi(J)\right)$,

(iii) $\operatorname{dist}\left(\Psi\left(\eta J^{+} \cup \eta J^{-}\right), \Psi(\partial D)\right)$,

(iv) $\operatorname{dist}(\Psi(J), \Psi(\partial D))$,

where by $\partial \Psi\left(\eta J^{+} \cup \eta J^{-}\right), \partial \Psi(J)$ we mean just the endpoints of these arcs. (The point of the cumbersome definition of $D_{z}$ is symmetry: if $z_{0}^{\prime}$ is the reflection of $z_{0}$ through $[|z|=s]$, then $D_{z_{0}^{\prime}}$ is the reflection of $D_{z_{0}}$ through $[|z|=s]$.)

If $\delta$ is small, then the following will be true:

A Brownian path (on $\mathbb{C}$ ) starting at a point $z_{0} \in D$ lying on one of the circles $[|z|=s],[|z|=s-\delta],\left[|z|=s+\frac{s}{s-\delta} \delta\right]$ will, with probability greater than $p$, hit an adjacent one of these circles before leaving $D_{z_{0}}$.

We will say a path on $D$ starting at a point $z_{0} \in \eta J^{+} \cup \eta J^{-}$completes a pass if it hits $[|z|=s]$ for the first time at a point $z_{1}$ without leaving $D_{z_{0}}$, and then hits $[|z|=s-\delta] \cup\left[|z|=s+\frac{s}{s-\delta} \delta\right]$ without leaving $D_{z_{1}}$. Note that by our conditions on $\sigma$, the point $z_{1}$ will be in $J$. If the path does this $m$ times in a row, we say it has completed $m$ successive passes. By our conditions on $\sigma$, if a path starts at a point $z_{0} \in \eta^{2} J^{+} \cup \eta^{2} J^{-}$and completes $m$ successive passes, then it will have stayed within $D$, all of its hits on $[|z|=s-\delta] \cup\left[|z|=s+\frac{s}{s-\delta} \delta\right]$ will have been on 
$\eta J^{+} \cup \eta J^{-}$, and all of its hits on $[|z|=s]$ will have been on $J$. Hence by (4.32) we see that:

$$
\begin{aligned}
& \text { A Brownian path on } D \text { starting at a point } \\
& z_{0} \in \eta^{2} J^{+} \cup \eta^{2} J^{-} \text {will, with probability greater than } p^{2 m} \text {, complete } \\
& m \text { successive passes. }
\end{aligned}
$$

We now wish to get an upper bound on the probability that a Brownian path on $D$ starting at a point $z_{0} \in \eta J^{+} \cup \eta J^{-}$will complete a pass and that at the time of completion of the pass, its lift to $\mathfrak{R}^{\prime}$ will still be on $D$. If $\delta$ is small enough and $N$ is large enough, our path will, with probability greater than $\frac{1}{3}$, hit $[|z|=s]$ for the first time at a point $z_{1} \in \bigcup_{j=0}^{N} I_{2 j+1}$, and do this without leaving $D_{z_{0}}$. Say it does this, and that $z_{1} \in I_{2 j_{1}+1}$. Let $\mathfrak{R}_{j_{1}}^{\prime}$ be the Riemann surface formed by attaching $\Delta_{j_{1}}$ to $D$ along $I_{2 j_{1}+1}$. By symmetry about $[|z|=s]$, if our path then hits $[|z|=s-\delta] \cup\left[|z|=s+\frac{s}{s-\delta} \delta\right]$ without leaving $D_{z_{1}}$, then at the time of this hit its lift to $\mathfrak{R}_{j_{1}}^{\prime}$ will be on $\Delta_{j_{1}}$ or $D$ with equal probability. However, it is not difficult to see that if a path on $D$ starting at $z_{0}$ completes a pass and if at the time of completion its lift to $\mathfrak{R}_{j_{1}}^{\prime}$ is on the sheet $\Delta_{j_{1}}$, then at this time its lift to $\mathfrak{R}^{\prime}$ will be on $\bigcup_{j=0}^{N} \Delta_{j}$ (but not necessarily on the particular sheet $\Delta_{j_{1}}$ ). Combining these observations, we see that:

The probability that a Brownian path on $D$ starting at a point $z_{0} \in \eta J^{+} \cup \eta J^{-}$will complete a pass, and that at the time of completion its lift to $\mathfrak{R}^{\prime}$ will still be on $D$, is less than $\frac{5}{6}$.

Combining (4.33), (4.34) and our other observations, we see that:

A Brownian path on $D$ starting at a point $z_{0} \in \eta^{2} J^{+} \cup \eta^{2} J^{-}$will, with probability greater than $p^{2 m}-\left(\frac{5}{6}\right)^{m}>1-\frac{1}{3} \tilde{\varepsilon}$, complete $m$ successive passes, at the time of completion of at least one of which its lift to $\mathfrak{R}^{\prime}$ will be on $\bigcup_{j=0}^{N} \Delta_{j}$.

This statement clearly implies that a Brownian path on $\mathfrak{R}^{\prime}$ starting on $\eta^{2} J^{+} \cup$ $\eta^{2} J^{-}$will hit $\left(\pi^{\prime}\right)^{-1}\left(\eta J^{+} \cup \eta J^{-}\right) \cap \bigcup_{j=0}^{N} \Delta_{j}$, where $\pi^{\prime}$ is the natural projection on $\mathfrak{R}^{\prime}$. To prove (4.31) it remains only to show that if $\delta$ is fixed and $N$ is made large enough, then for every $j=0,1, \ldots, N$, a Brownian path on $\mathfrak{R}^{\prime}$ starting at a point on $\left(\pi^{\prime}\right)^{-1}\left(\eta J^{+} \cup \eta J^{-}\right) \cap \Delta_{j}$ will, with probability greater than $1-\frac{1}{3} \tilde{\varepsilon}$, hit $\partial \Delta_{j}$. This however is obvious, since $\left|I_{j}\right|=\frac{1}{2 N+1}|J|$. This completes Step 2. Hence Theorem 2 is proved.

\section{ACKNOWLEDGMENTS}

I am very grateful to John B. Conway for showing me the connection between Rudin's question and induced measures. I have had useful conversations about induced measures with Chris Bishop, Dan Luecking, Joel Shapiro, and Ken Stephenson, and I am grateful to all of them.

\section{REFERENCES}

[ABR] Axler, S., Bourdon, P. and Ramey, W., Harmonic Function Theory. Springer-Verlag, New York-Berlin, 1992. MR 93f:31001 
[AS] Ahlfors, L. V. and Sario, L., Riemann Surfaces. Princeton University Press, Princeton, New Jersey, 1960. MR 22:5729

[B] Bourdon, P. S., Rudin's orthogonality problem and the Nevanlinna counting function. Proc. Amer. Math. Soc 125, 4(1997), 1187-1192. MR 98b:30034

[C1] Carleson, L., An interpolation theorem for bounded analytic functions. Amer. J. Math 80 (1958) 921-930. MR 32:8129

[C2] Carleson, L., Interpolations by bounded analytic functions and the corona problem. Ann. of Math., 76 (1962), 547-559. MR 25:5186

[C] Conway, J. B., Functions of one complex variable. Springer-Verlag, New York-Berlin, 1978. MR 80c:30003

[D] Duren, P. L., Theory of $H^{p}$ spaces. Academic Press, New York, 1970. MR 42:3552

[F] Fisher, S. D., Function Theory on Planar Domains. John Wiley and Sons, New York, 1983. MR 85d:30001

[G] Garnett, J. B., Bounded Analytic Functions. Academic Press, New York, 1981. MR 83g:30037

[K] Kakutani, S., Two-dimensional Brownian motion and harmonic functions. Proc. Imp. Acad. Tokyo, 20 (1944), 706-714. MR 7:315b

[Ke] Kellogg, O. D., Foundations of Potential Theory. Dover, New York, 1953. MR 36:5369

[L] Littlewood, J. E., On inequalities in the theory of functions. Proc. London Math. Soc. (2) 23 (1925), 481-519.

[P] Petersen, K. E., Brownian Motion, Hardy Spaces, and Bounded Mean Oscillation. Cambridge University Press, Cambridge, 1977. MR 58:31383

[PS] Port, S. C. and Stone, C. J., Brownian Motion and Classical Potential Theory. Academic Press, New York, 1978. MR 58:11459

[R] Rudin, W., A generalization of a theorem of Frostman, Math. Scand., 21 (1967), 136-143. MR 38:3463

[S] Shapiro, J. H., The essential norm of a composition operator. Ann. of Math., 125 (1987), 375-404. MR 88c:47058

[Sp] Springer, G., Introduction to Riemann Surfaces. Addison-Wesley, Reading, Massachusetts, 1957. MR 19:1169g

Department of Mathematics, University of Tennessee, Knoxville, Tennessee 379961300

E-mail address: sundberg@math.utk.edu 